\title{
Removal of formaldehyde and xylene fumes from histopathology laboratories: a functional approach to the design of extraction systems
}

\author{
FP EDWARDS, AR CAMPBELL
}

\section{From the Department of Histopathology, North Manchester General Hospital, Crumpsall, Manchester M8 6RB}

SUMMARY There have been many reports of the relative hazards of formaldehyde, xylene, and hot paraffin wax fumes in pathology laboratories. In contrast, there have been comparatively few describing efficient extraction systems for their removal. When the opportunity presented to design a new laboratory the efficient removal of these fumes was given a high priority. In designing the extraction systems described consideration was given to the physical properties of the fumes and the degree of freedom of use of the bench, with the object of achieving sub-threshold limit values for each area of exposure. The efficiency of removal of the formaldehyde and xylene was measured using infrared spectroscopy.

Increasing awareness of the health hazards associated with chemicals commonly used in histology departments has stimulated both equipment manufacturers and medical laboratory scientists to improve the laboratory environment by reducing the release of toxic fumes. Closed circuit tissue processors and the absorption of fumes by charcoal filters are both examples of developments by manufacturers.

The installation of an extraction system in a laboratory is not easy for a variety of reasons. The installation is usually undertaken only when a new laboratory is built or when an existing block is converted. The latter may well present difficulties in installing extraction. The unavailability of proven systems and often the lack of liaison between the laboratory staff and planning team can result in the installation of an inefficient system.

In 1982 the histopathology department at North Manchester General Hospital moved into a new laboratory which was formerly a ward. The extraction system installed was based on the original concepts proposed by the laboratory staff. These often rudimentary ideas were developed by the architect and heating consultant to give an extraction system which was not only very efficient but designed with the user in mind.

\section{Design objectives}

Three basic objectives were set:

1 To achieve sub-threshold limit values for each Accepted for publication 20 December 1983 chemical used in the laboratory.

2 To keep each working area free from obstructions such as hoods, canopies, cabinets.

3 To remove the traditional odours associated with histology laboratories.

A balanced warmed air heating system was chosen to provide the general ventilation. This provides filtered warm air to the laboratory at four to six air changes each hour. The system is supplemented by individual local extraction modules built into each bench where toxic agents are used. These are connected directly to the external environment at the second storey level of the building.

The design considerations for the extraction modules were:

1 Threshold limit value of the agent. Each module must not exceed, under normal working conditions, the figures of $2 \mathrm{ppm}$ for formaldehyde and $100 \mathrm{ppm}$ for xylene. These figures were used as maximum exposure limits not as time weighted averages.

2 Accessibility to the bench. Where an area is constantly used or the whole depth of the bench is needed or where articles are often moved on or off the bench the extraction must be achieved without hindrance to the worker. Bench areas considered to be in this category were: specimen dissection (cut up), tissue disposal, wax embedding, staining, and slide mounting. Areas where total freedom of access was not necessary were: tissue processing, staining machines, and fixative preparation.

3 Physical characteristics of the toxic vapour. (a) Inflammability-fans with spark proof motors 
are essential when exhausting xylene. Installing fans with motors outside the air current was an added precaution. (b) Vapour density - the direction of extraction was a prime consideration. Removal of the agent away from the worker providing the greatest protection. Consideration of the density of the agent and its extraction along the direction of its natural path will give more efficient extraction and enhance protection. Formaldehyde being only slightly heavier than air, with a relative vapour density of 1.07 , tends to remain static at the liberation point, while xylene (relative vapour density 3.67 ) will fall naturally. The capture velocity necessary will vary according to the density of the agent.

\section{Formaldehyde extraction}

Four areas were identified where contact with formaldehyde was such that local extraction was necessary.

\section{SPECIMEN DISSECTION AREA}

This consists of a one piece stainless steel unit $2000 \times$ $600 \mathrm{~mm}$ available commercially as a Grantham Slop Hopper unit (Dent \& Hellyer Ltd, Wallworth Road, Andover, Hants). It incorporates a flat dissection area, $800 \times 480 \mathrm{~mm}$, a central sink, $520 \times 400 \times$ $220 \mathrm{~mm}$, and a sluice, face opening $350 \mathrm{~mm}$ diameter (Fig. 1). The unit has been modified to accommodate a sparge pipe to supply a cleansing sheet of water over the dissection area. Also fitted is a hand held low pressure water spray, which is used to cleanse a raised Corian (Corian Distribution, Richmond House, Blenheim Terrace, Leeds) cutting table $500 \times 360 \mathrm{~mm}$, sited on the dissection area. The sink is used to open fluid filled cysts and so on, and the sluice to open and clean lengths of bowel.

Removal of formaldehyde is through a single extract slot, $22 \times 1880 \mathrm{~mm}$, raised $40 \mathrm{~mm}$ over the bench surface. The extract slot runs the full length of the unit immediately behind all three work areas. The point of extraction can be altered by using the valves in the trunking (Fig. 2) and any combination of all three areas can be ventilated. The fan is sited beneath the unit and has a variable speed control. Air flow measurements using a hot wire anemometer at a point $25 \mathrm{~mm}$ in front of the extract slot gave the figures shown in the Table.

Removal of the formaldehyde is from front to back, the raised cutting table allowing air to pass under as well as over the specimen area. The dissection area is regularly irrigated using the hand spray and sparge pipe to remove residual fixative.

The efficiency of the extraction system was measured by monitoring formaldehyde concentrations using a Miran (Wilkes Scientific Co Ltd, 64 Burners Lane, Kiln Farm, Milton Keynes) infrared gas analyser at wavelength $3.58 \mu \mathrm{m}$ coupled to a flat bed chart recorder. Air was sampled at chest height continuously through two typical cut up sessions conducted by the same pathologist. Fig. 3 shows the formaldehyde concentrations reached without extrac-

Airflow velocity measurements for the constituent areas of the dissection bench

\begin{tabular}{lll}
\hline Air flow velocity $(\mathrm{m} / \mathrm{s})$ & \\
\hline Sluice & Sink & Cut up area \\
\hline $1.778 \mathrm{~m} / \mathrm{s}$ & $2.032 \mathrm{~m} / \mathrm{s}$ & $2.79 \mathrm{~m} / \mathrm{s}$ \\
Vent closed & $2.54 \mathrm{~m} / \mathrm{s}$ & $3.302 \mathrm{~m} / \mathrm{s}$ \\
Vent closed & Vent closed & $4.064 \mathrm{~m} / \mathrm{s}$ \\
\hline
\end{tabular}

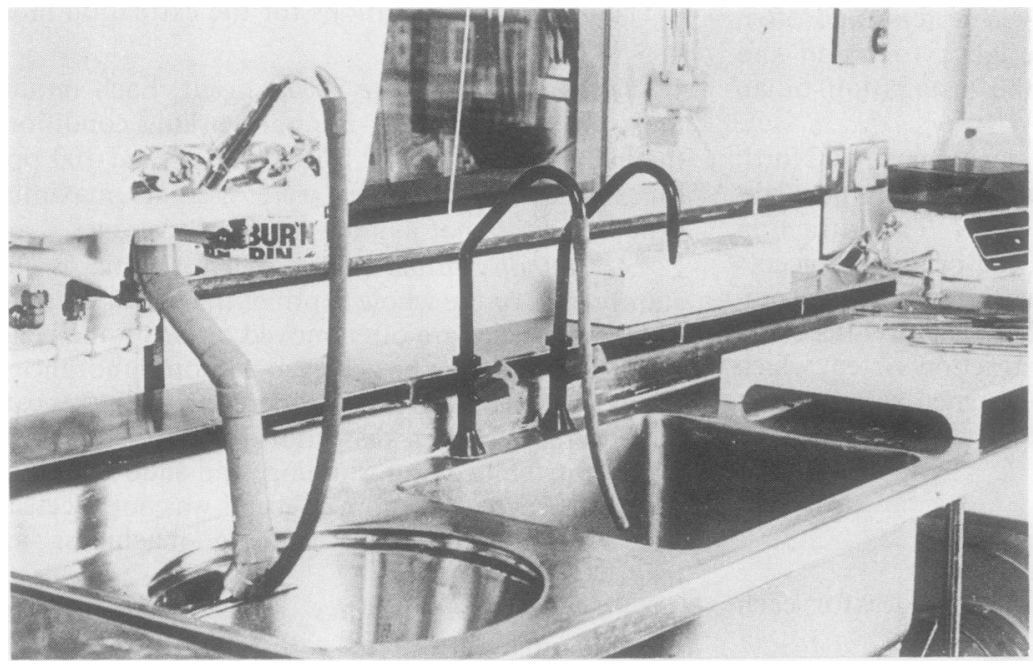

Fig. 1 Specimen dissection area showing cut up area, sink, and sluice. 


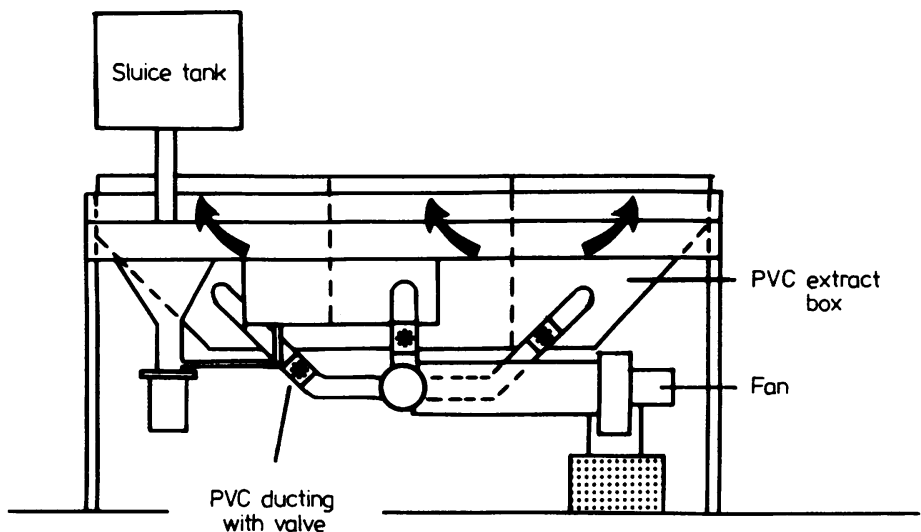

Fig. 2 Specimen dissection unit showing details of construction of extraction trunking and fan. Note valves in trunking to control point of extraction.

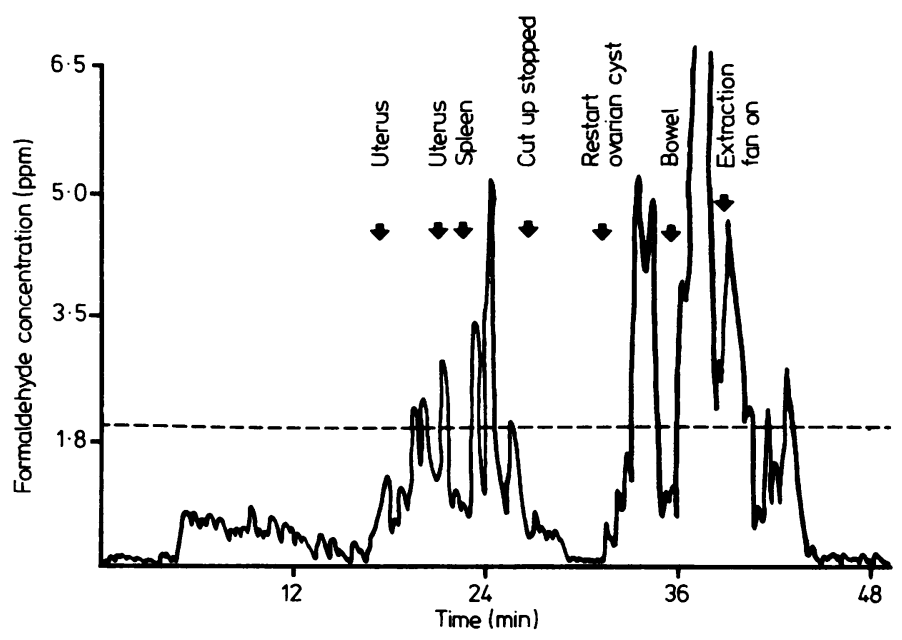

Fig. 3 Formaldehyde concentrations during a cut up session with no extraction. The fan was switched on after about $40 \mathrm{~min}$ when concentrations reached $13 \mathrm{ppm}$ (off scale).

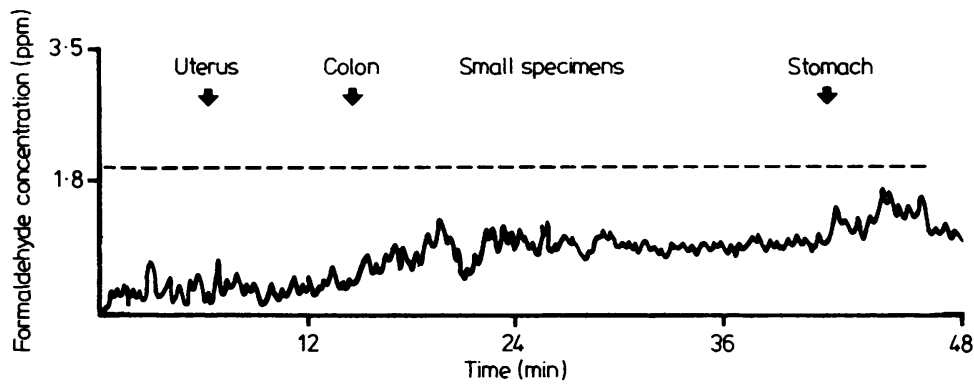

Fig. 4 Formaldehyde concentrations during a cut up session with extraction fan on.

tion and Fig. 4 shows values reached when the fan was on throughout the cut up session.

Noise level from the unit was $57 \mathrm{dBA}$, which is not considered excessive (threshold limit value $90 \mathrm{dBA}$ ). This level does not prohibit dictation of specimen descriptions to secretarial personnel or tape recorder.
SPECIMEN DISPOSAL

The formaldehyde released during tissue disposal and washing of specimen pots is removed by extraction vents sited above and to the rear of the disposal machine hopper (Haigh Tissue Disposal Machine, Model 55B, Haigh Engineering Co Ltd, Alton Road, 


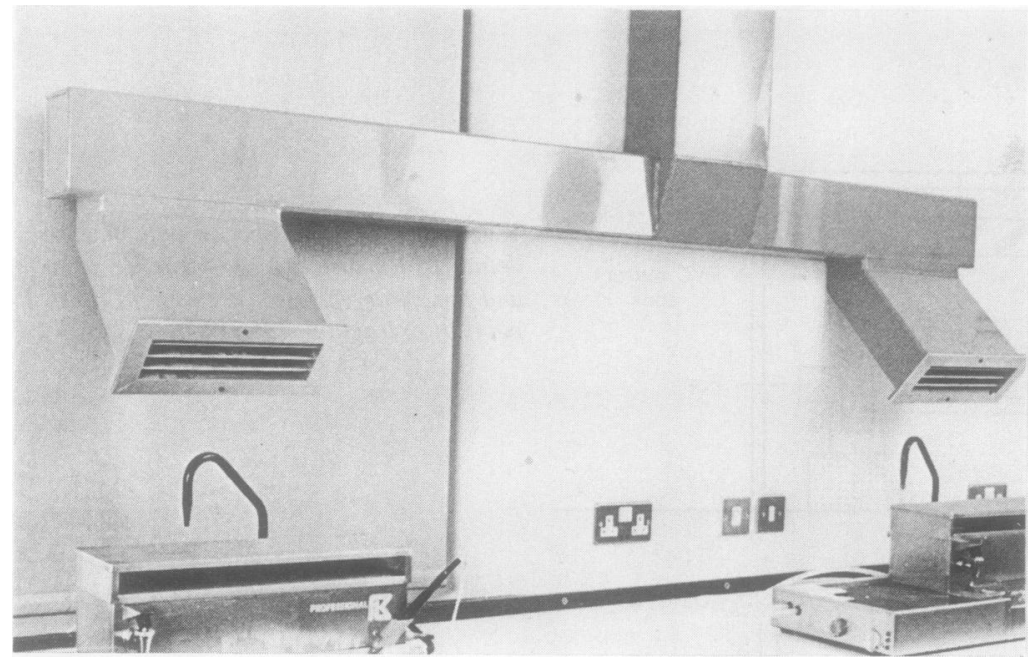

Fig. 5 Extraction vents and ducting over wax embedding units.

Ross on Wye) and the wash up sink. The vents, sited $600 \mathrm{~mm}$ over the washing areas, are angled at $45^{\circ}$ to improve vapour capture. Air flow velocity through the vents is $1.016 \mathrm{~m} / \mathrm{s}$. Formaldehyde concentrations

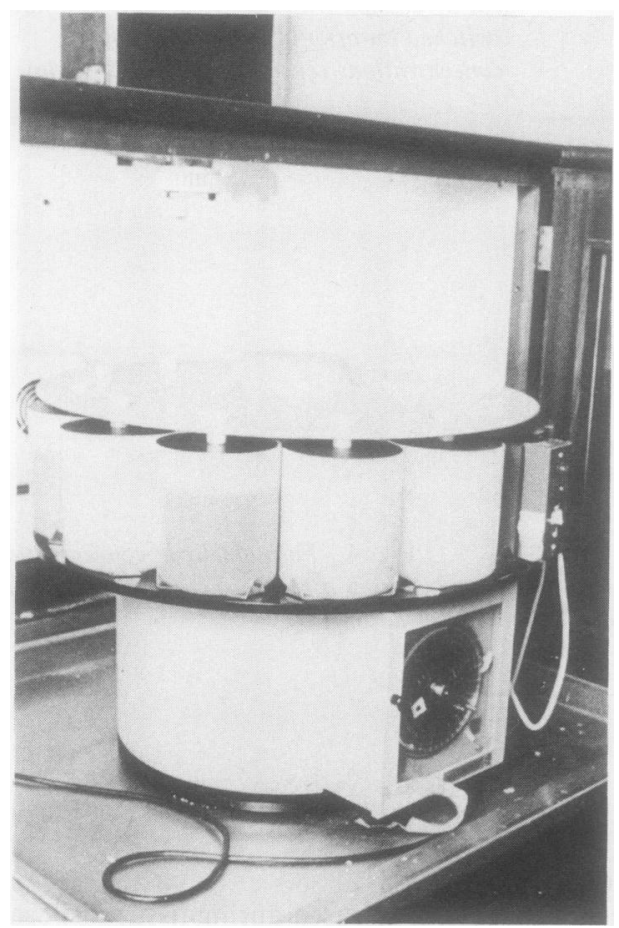

Fig. 6 Tissue processor housed in extraction cupboards underneath the benches and mounted on mobile platforms. The extraction fans can be seen on the back wall and smoke sensor on roof of cupboard. monitored throughout an entire tissue disposal session indicated that the system maintains formaldehyde concentrations below the threshold limit value at all times.

\section{FORMALIN FIXATIVE PREPARATION}

A closed circuit pumped system was constructed to prepare the large quantities of formalin fixative ${ }^{1}$ needed to supply several hospitals and postmortem examination rooms. The apparatus consists of a 1251 closed polythene tank. Mounted over this, on a gantry, are two peristaltic pumps, a paddle mixer, and a water inlet solenoid valve controlled by an automatic fluid level detector. The whole unit is housed in a cupboard from which air is constantly extracted at a rate of $0.638 \mathrm{~m}^{3} / \mathrm{s}$. This system enables $120 \mathrm{l}$ of fixative to be prepared and dispensed without contaminating the laboratory atmosphere.

\section{STORAGE OF SPECIMENS}

Specimens stored in polythene containers on open shelves can contribute to the general concentration of formaldehyde in the atmosphere. To remove this source of contamination all specimens are kept in cupboards which have constant extraction at $0.319 \mathrm{~m}^{3} / \mathrm{s}$. The shelves are perforated to allow air movement within the cupboard. Opening the cupboard doors for a few minutes produces a concentration of formaldehyde of $1.4 \mathrm{ppm}$ in the laboratory adjacent to the cupboard.

\section{Paraffin wax fume extraction}

The hazards associated with paraffin wax fumes are largely unidentified as the composition of the fumes can vary with the wax constituents, additives, 


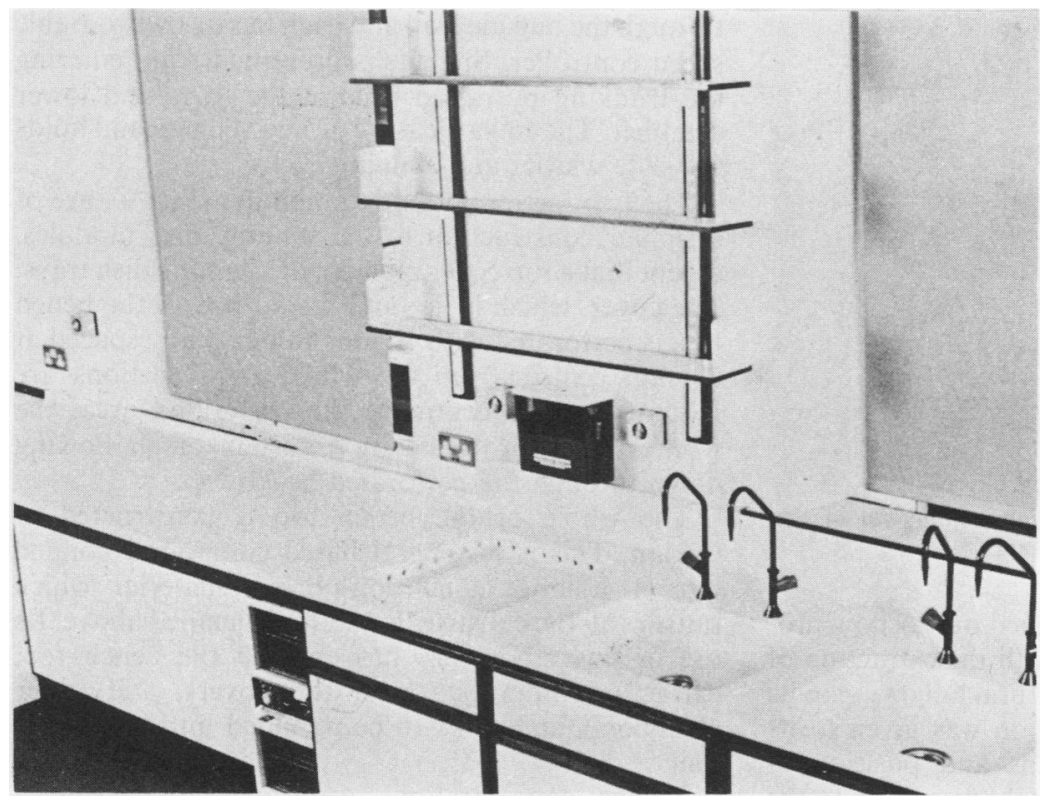

Fig. 7 Staining and section mounting area composed of two mounting modules, pre- and poststaining dish modules on either side of the sinks. The individual fan speed controllers are mounted on the rear wall over each unit.

impurities, solvents, and also the degree of heating. In recognition of the possible dangers we decided to remove paraffin wax fumes from the laboratory.

\section{EMBEDDING CENTRES}

Most of the fumes generated from embedding centres come from the hotplate and wax reservoir area. They rise in the hot air and are captured by siting angled extraction vents $200 \mathrm{~mm}$ directly over the hotplate and reservoir area (Fig. 5). The air flow is $2.032 \mathrm{~m} / \mathrm{s}$ through the vents to provide complete capture as shown by smoke pencil tests.

\section{TISSUE PROCESSORS (CAROUSEL TYPE)}

Here, in addition to hot wax, formalin and xylene have to be considered. The mixture of fumes of varying vapour density, the flammability of the reagents, and the physical size and complexity of the machines make efficient fume extraction by air flow alone an impossibility. To enclose the processor is the only practical solution if the problem of accessibility can be solved. To achieve this, and to save space in the laboratory, the processors have been housed in cupboards under the benches (Fig. 6). These have constant extraction at $0.319 \mathrm{~m}^{3} / \mathrm{s}$, alarm protected fans, and heat and smoke sensors. The processors stand in drip trays mounted on runners, allowing them to be pulled out smoothly for cleaning and changing; loading and programming can be done without moving the processors.

\section{Xylene vapour extraction}

Protection against xylene fumes was necessary for the manual staining bench, the section mounting area, and around the two staining machines.

MANUAL STAINING AND SECTION MOUNTING

These two areas were combined as both demanded a similar degree of freedom on the bench-thus an

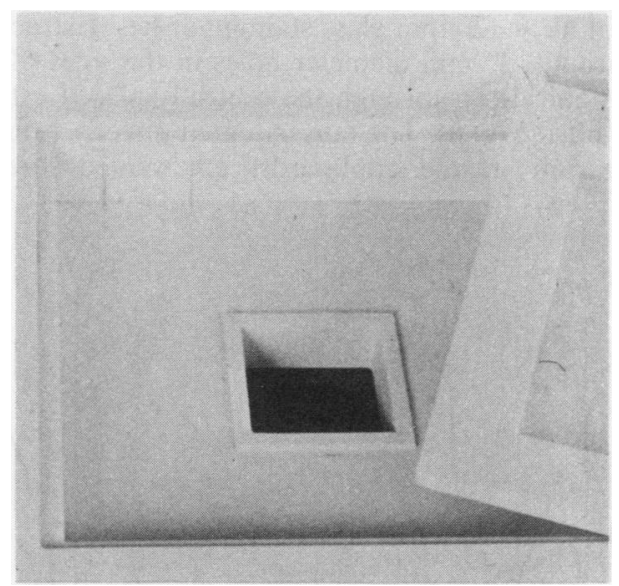

Fig. 8 A staining dish extraction module with tray partially removed to show lower chamber construction and extract port position. Note rim around port to contain spillage. 


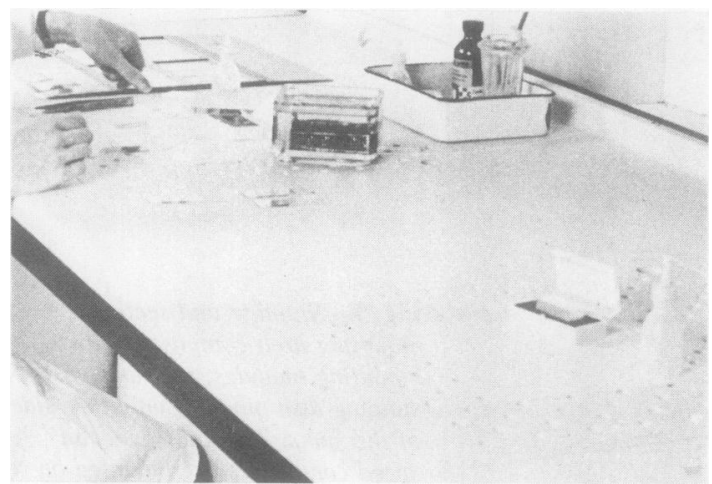

Fig. 9 Perforated bench top extraction modules for section mounting.

enclosed extraction system was ruled out. A flow protection system was decided on, with the extraction of xylene downwards aided by its natural fall owing to its high vapour density. Due attention was given to its flammability in the type of fans and position of switchgear and so on. Much thought was given to the choice of material for the bench construction. Resistance to the commonly used staining reagents and to delamination by water together with the ability to construct the extraction modules and sinks without leakage problems were all important considerations.

The staining and section mounting bench is a one piece unit measuring $5520 \times 750 \mathrm{~mm}$. It incorporates four down draught extraction modules and two sinks moulded into the bench (Fig. 7). The modules either side of the sinks are trays sunk into the bench top to hold the xylene and alcohol dishes before and after staining. The trays measure $450 \times 450 \mathrm{~mm}$ and hold nine $120 \times 120 \mathrm{~mm}$ glass staining dishes. Extraction is through $20 \mathrm{~mm}$ diameter holes in the sides of the trays and down through the centrally placed extraction port (Fig. 8). The fans, situated directly beneath each unit in the cupboards, are vented directly through the outside wall and each has its own variable speed controller. Spillage is prevented from entering the trunking by raised rims in the trays and lower chamber. The sinks measure $525 \times 400 \mathrm{~mm}$ and holds the slide washer and staining racks.

The two section mounting modules (Fig. 9), are of a similar construction to the staining dish modules, except that a top cover replaces the staining dish trays. The cover, which is flat and fits flush with the bench top, is perforated with $10 \mathrm{~mm}$ holes equally spaced at $33 \mathrm{~mm}$ centres over the whole area. Sections are mounted at the centre of the perforated area, the worker being protected by a curtain of air flowing down through the perforated bench top.

The whole of the bench top is constructed of Corian. This is a mineral based compound bonded into a homogeneous non-porous material which satisfies all the construction criteria detailed above. Its use in this laboratory has enabled the bench top, extraction modules (trays, top covers, and lower chambers), and sinks to be moulded into a jointless unit.

Xylene concentrations were measured using a Miran gas analyser at wavelength $13 \cdot 19 \mu \mathrm{m}$. The sample point was $150 \mathrm{~mm}$ over the xylene dishes and midway between the person's face and the slide being mounted. Xylene concentrations over the dishes before and after staining increased rapidly in still air when the covers were removed. Within two and a half minutes of switching on the fans, xylene concentrations fell from $75 \mathrm{ppm}$ to nearly zero (Fig. 10). Extraction rates of similar efficiency were achieved through the section mounting modules. A figure of under $10 \mathrm{ppm}$ was achieved with the fan on compared with 75 ppm with no extraction (Fig. 11).

\section{STAINING MACHINES}

The intermittent use of the staining machines used in the laboratory determined that enclosure extraction was adopted.

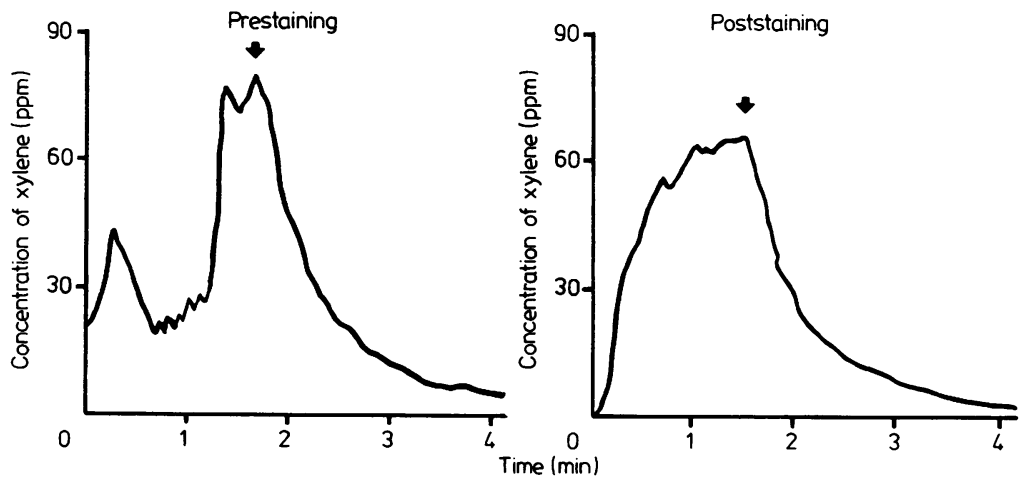

Fig. 10 Xylene concentration over the dishes. The glass lids were removed at the start of the trace and extraction fans switched on after 2 min (arrow's). 


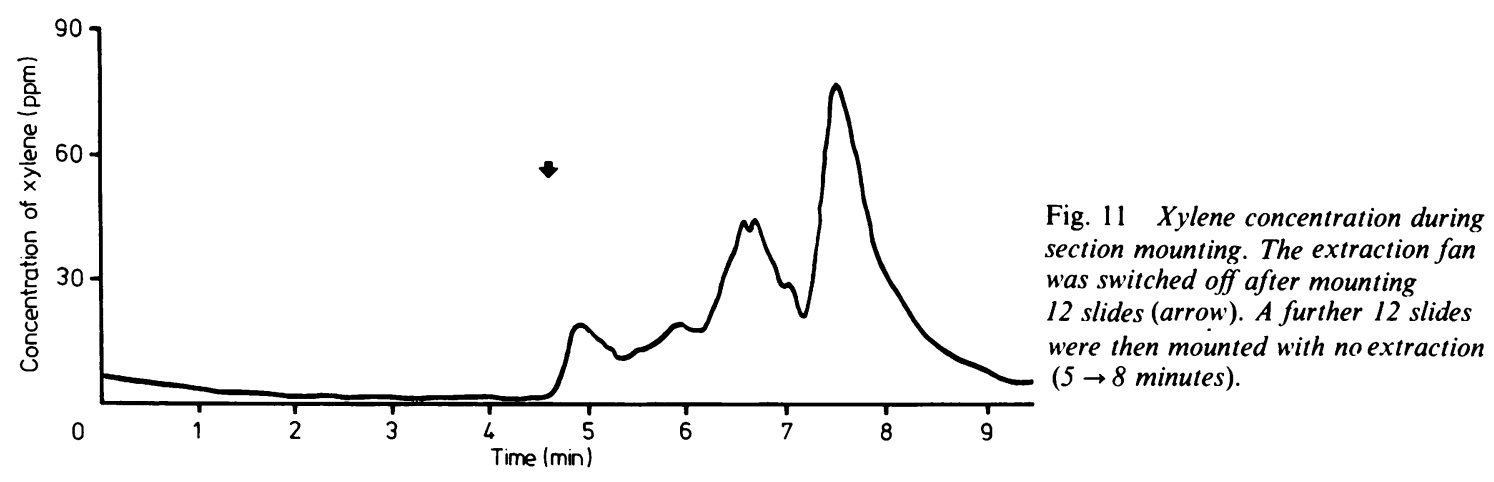

\section{Carousel type machines}

To contain and remove xylene and alcohol vapour emanating from a Shandon 23 stage stainer, used for Papanicolaou staining, a free standing perspex cover $760 \times 740 \times 680 \mathrm{~mm}$ was provided. The front is detachable and has a slot at its base to allow air entry. The cover is shaped to fit the contour of the back wall, through which an extraction fan removes the fumes. The cabinet size is large enough to permit daily machine operation and light enough to be removed while cleaning the machine and changing reagents. The fan is kept running during these operations.

\section{Linear staining machine}

Owing to the location of the SKI linear stainer in the laboratory and the accessibility needed, it was impossible to extract this equipment without unsightly trunking and loss of access. A Shandon Fumeguard (Shandon Southern Products Ltd, Astmoor, Runcorn) with its xylene filtration system was purchased. Preliminary tests indicate a poor level of extraction with appreciable escape of xylene from the reservoir end of the machine. The position of the extract fans relative to the reservoir is the main reason for the failure to capture a vapour as heavy as xylene. An improved model (Shandon Linistain Retro Fume Hood) has recently been introduced featuring a more compact design.

\section{Discussion}

The Health and Safety Executive and its American equivalent, the National Institute for Occupational Safety and Health, both present a formidable array of references to published work on the health hazards of formaldehyde. ${ }^{23}$ These include acute toxicity in a wide range of experimental animals, ${ }^{4-6}$ eye irritancy, ${ }^{7}$ skin sensitisation, ${ }^{8}$ some evidence of mutagenic effect, ${ }^{910}$ and carcinogenicity in rats and mice. ${ }^{1112}$ In man there are reported acute toxic effects due to ingestion ${ }^{13}$ and inhalation, ${ }^{14}$ skin $^{15}$ and eye ${ }^{16}$ irritancy, and skin ${ }^{17}$ and possibly lung ${ }^{18}$ sensitisation.

There is no evidence that exposure to formaldehyde has produced cancer in humans. The weight of experimental evidence is such, however, that the American Institute has recommended that although humans and animals may differ in their susceptibility to specific chemical compounds, any substance that produces cancer in animals should be considered a risk to humans. ${ }^{3}$

The hazards associated with the use of xylene are not as extensively reported. They include acute respiratory distress in humans, ${ }^{19}$ but xylene is given a moderate toxicity rating, with headache, nausea, loss of appetite, lassitude, and impairment of coordination all being quoted. ${ }^{20}$

Faced with the considerable range of effects from these two commonly used reagents in our laboratory, the senior staff were determined to provide a safe working environment. The initial step in our design was mentally to enclose each bench operation and its contaminant and then make such openings or changes in the enclosure as were dictated by the operation therein. The air flow then compensates for the openings and design compromises. We soon realised that so much access was needed for the specimen cut up, tissue disposal, and staining and section mounting benches that enclosure by hoods or cabinets was impracticable as well as undesirable for the desired clean design of the laboratory. The only alternative was to provide protection for the staff by removing the contaminant by efficient capture using air flow alone. The success of this depends on the efficiency of the system and is achieved in three main ways. Firstly, by way of the forced air heating system to provide general ventilation. This also provides slight positive air pressure in the laboratory which ensures each local extraction module is never starved of air. Secondly, noting the density of the fumes, it was proposed to use the direction of their natural flow. More efficient capture can then be achieved with lower air velocities. Finally, each extraction vent was positioned as near as possible to the contaminant point compatible with the 
freedom of movement on the bench.

The resultant local extraction modules described are very efficient as can be seen by the gas analyser traces. At no time during normal working are the threshold limit values for formaldehyde or xylene exceeded during specimen cut up, tissue disposal, or section staining and mounting. In fact these figures are achieved with the fan speeds set below their maximum values. This gives latitude to deal with abnormal loads (gross spillage) or any future reduction in threshold limit values. As the efficiency and air velocities for each module on installation are known, these can be measured yearly to monitor the performance of the systems.

The complexity of tissue processors and staining machines makes the design of an open air flow extraction system virtually impossible. The limited need for accessibility and the fact that when spillage occurs liquids may enter the equipment, making cleaning difficult, prompted us to choose enclosure type extraction for the carousel tissue processors and staining machines. The need to reach the rear of the equipment for cleaning and reagent replacement was met in the case of the processors by placing them on mobile platforms; for the staining machine a cabinet light enough to be easily removed was constructed.

Experience in using the Corian staining bench for the past year has endorsed the decision to use this material. Its excellent moulding and jointing properties have resulted in a smooth leak free unit. Its relative resistance to stains keeps cleaning to a minimum and surface damage, should it occur, can be removed by simple abrasives. The designs have given complete freedom of use and when not in use for section mounting the area is available for general use.

By considering the ergonomic requirements, the freedom of movement needed, and the physical properties of the fumes liberated modular extraction units have been designed which impose few restrictions and difficulties in their use. This has given a more efficient laboratory in which the staff benefit from a safer working environment.

We acknowledge the invaluable help of Mr Barry Ransom and Mr Roy Evans, members of the planning team, Dr D deKretser and Mr DJ Jenkins for their assistance in the preparation of this manuscript, and Miss J Perry for the photographic assistance.

\section{References}

${ }^{1}$ Carson FL, Martin JH, Lynn JA. Formalin fixation for electron microscopy. A re-evaluation. Am J Clin Pathol 1973;59:365-73.

${ }^{2}$ Health and Safety Executive. Toxicity review, 2: Formaldehyde. London: HMSO, 1981.

${ }^{3}$ Robbins A, Bingham E. Formaldehyde: evidence of carcinogenicity. NIOSH/OSHA, Current Intelligence Bulletin 34, Cincinnati, Ohio: NIOSH Publications, 1980.

${ }^{4}$ Salem $\mathrm{H}$, Cullumbine $\mathrm{H}$. Inhalation toxicities of some aldehydes. Toxicol Appl Pharmacol 1960;2:183-7.

${ }^{5}$ Kilburn KH, McKenzie WN, Thurston RJ. Effect of formaldehyde as vapour and absorbed on carbon, on hamster airways. Am Rev Resp Dis 1976;113:238.

${ }^{6}$ Feldman YG, Bonashevskaya TI. On the effects of low concentrations of formaldehyde. Hygiene and Sanitation 1971;36: 174-80.

${ }^{7}$ Grant WM. Toxicology of the eye. 2nd ed. Springfield, Illinois: Charles C Thomas, 1974:502-7.

${ }^{8}$ Maurer Th, Thomann P, Weirich EG, Hess R. Predictive evaluation in animals of the contact allergenic potential of medically important substances. II Comparisons of different methods of cutaneous sensitisation with weak allergens. Contact Dermatitis 1979;5:1-10.

${ }^{9}$ Rosenkranz HS. Formaldehyde as a possible carcinogen. Bull Environ Contam Toxicol 1972;8:242-4.

${ }^{10}$ Wilkins RJ, Macleod HD. Formaldehyde induced DNA-protein crosslinks in Escherichia coli. Mutat Res 1976;36:11-6.

11 Kerns WD. Long term inhalation toxicity and carcinogenicity studies of formaldehyde in rats and mice. Reported at the third CIIT conference on toxicology. Raleigh, North Carolina: 1980.

${ }_{12}$ Swenberg JA, Kerns WD, Mitchell RI, Gralla EJ, Pavkov KL. Induction of squamous cell carcinomas of the rat nasal cavity by inhalation exposure to formaldehyde vapour. Cancer Res 1980;40:398-402.

${ }^{13}$ Rathery F, Piedelieure R, Delarue J. Death by absorption of formalin. Annales de Medicine Legale, de Criminologie et de Police Scientifique. 1940;20:201-9.

${ }^{14}$ Fassett DW. Aldehydes and acetals. In: Patty FA, ed. Industrial hygiene and toxicology. 2nd ed. New York: Interscience, 1963:1970-2.

15 Proctor NH, Hughes JP. Chemical hazards in the workplace. Philadelphia: Lippincott, 1978;272-4.

${ }^{16} \mathrm{Kelecom} \mathrm{J}$. Corrosive eye damage with formalin. Arch Ophthalmol (Paris) 1962;22:259-62.

${ }^{17}$ Marzulli FN, Maibach HI. The use of graded concentrations in studying skin sensitives: Experimental contact sensitisation in man. Food Cosmet Toxicol 1974;12:219-27.

${ }^{18}$ Sakula A. Formalin asthma in hospital laboratory staff. Lancet 1975;ii:816.

${ }^{19}$ Bush CL, Nelson CE. Xylene (a warning of its use in the histology and cytology laboratory). Medicine 1977;8:16.

${ }^{20}$ Sax NI. Dangerous properties of industrial materials. 5th ed. Van Rostrand Rheinhold: New York, 1979:1094-5

Requests for reprints to: Mr FP Edwards, Department of Histopathology, North Manchester General Hospital, Crumpsall, Manchester M8 6RB, England. 